\title{
La violencia física, psicológica, emocional y sexual durante el embarazo: riesgo reproductivo predictor de bajo peso al nacer en Costa Rica
}

\author{
Hilda Patricia Núñez-Rivas, ${ }^{1}$ Rafael Monge-Rojas, ${ }^{1}$ Carla Gríos-Dávila, ${ }^{2}$ \\ Ana María Elizondo-Ureña ${ }^{1}$ y Ana Rojas-Chavarría ${ }^{3}$
}

RESUMEN Objetivo. Determinar la prevalencia de la violencia física, psicológica, emocional y sexual durante el embarazo y su asociación con el bajo peso al nacer.

Métodos. Se exploró la violencia sufrida por 118 embarazadas de la comunidad urbana marginal Finca San Juan, de Rincón Grande de Pavas, San José, Costa Rica, que dieron a luz entre septiembre de 1998 y noviembre de 1999. Se utilizó un cuestionario de preguntas cerradas previamente validado. Mediante un modelo de regresión lineal múltiple se ajustaron las diferencias entre las medias del peso de los recién nacidos según las características de la madre (edad, años de estudio, condición conyugal, deseo del embarazo, hábitos nocivos, número de embarazos y partos previos, intervalo intergenésico, estatura, aumento total de peso durante el embarazo y enfermedades durante la gestación). Se utilizó un modelo de regresión logística para medir el efecto directo de la violencia sobre el bajo peso al nacer, así como un método no paramétrico para calcular la fracción atribuible en las mujeres expuestas.

Resultados. Los niños de madres que sufrieron actos de violencia pesaron como promedio $449,4 \mathrm{~g}$ menos $(\mathrm{P}<0,001)$ que los de las mujeres que no habían estado expuestas a actos de violencia. Las primeras presentaron un riesgo tres veces mayor de tener hijos con bajo peso al nacer que las segundas (IC95\%: 1,39 a 8,10). Las variables que se asociaron más estrechamente con el bajo peso al nacer fueron la violencia sufrida por la madre (asociación directa) y el aumento de peso de la madre durante la gestación (relación inversa).

Conclusiones. Los resultados obtenidos indican la necesidad de investigar el tema más profundamente, instruir al personal de salud sobre la violencia hacia las mujeres como factor de riesgo reproductivo y conformar grupos de expertos en este tema con el fin de desarrollar protocolos especializados para la identificación temprana de embarazadas sometidas a algún tipo de agresión.

Palabras clave Violencia doméstica, mujeres maltratadas, mujeres embarazadas, recién nacido de bajo peso, Costa Rica.

Instituto Costarricense de Investigación y Enseñanza en Nutrición y Salud (INCIENSA), Ministerio de Salud, San José, Costa Rica. Dirigir la correspondencia a: Hilda P. Núñez-Rivas, INCIENSA, Apdo. 4, Tres Ríos, Costa Rica. Teléfono: (506) 279-9911; fax: (506) 279-5546; correo electrónico: hnunez@inciensa.sa.cr

2 Caja Costarricense de Seguro Social, San José, Costa Rica.
La violencia hacia las mujeres es un problema poco reconocido por los diferentes sectores vinculados con la salud humana - los de la salud, educación y trabajo, entre otros- e insuficientemente valorado como una prioridad de la salud

Instituto Nacional de la Mujer (INAMU), San José, Costa Rica. pública (1), a pesar de que es una causa importante y frecuente de morbilidad y mortalidad femeninas (2-4) y de que puede representar la pérdida de uno de cada cinco días de vida saludable entre mujeres en edad reproductiva $(3,5)$.

La violencia hacia las mujeres embarazadas repercute en los recién nacidos, pues conduce al bajo peso al nacer (BPN) y afecta adversamente 
acualquierniñoenlaetapainicial dela vida, tanto por el incremento de la morbilidad y de la mortalidad como por sus efectos sobre el desarrollodelascapacidadesfísicas, cognoscitivas y sociales, que pueden limitarconsiderablemente a los que sobreviven estos episodios (6-10).

Este tipo de violencia se caracteriza por un patrón de conducta coercitiva hacia las mujeres que abarca el abuso físico (golpes, quemaduras, mordeduras, heridas con arma blanca o de fuego), el abuso psicológico y emocional (intimidación, humillaciones verbales, manipulación, omisión, abandono, negligencia) y el abuso sexual, el cual consiste en forzar física o psicológicamente a la mujer a la relación sexual o a una conducta sexual de determinado tipo (11).

La información sobre la violencia hacia las mujeres es escasa y varía según el diseño de los estudios y los países donde estos se realizan. En una revisión de 26 trabajos procedentes de 20 países realizada por Heise (5), entre 20 y $75 \%$ de las mujeres habían sufrido actos de violencia. El estudio reveló grandes diferencias no solo según el país, sino también según el tipo de población estudiada (urbana o rural). En un metaanálisis de ocho estudios, Murphy et al. (12) encontraron prevalencias de abuso durante el embarazo entre 5,6 y 16,6\%.

Hasta la fecha, pocas investigaciones realizadas en Costa Rica han examinado el efecto de factores psicosociales durante el embarazo en el peso de los recién nacidos. Los principales hallazgos han sido la asociación entre el BPN y la violencia, así como el deseo del embarazo, la sensación de seguridad de la embarazada y el apoyo y la compañía durante la gestación, como factores protectores $(8,11)$.

La violencia hacia una mujer embarazada puede tener graves consecuencias para la madre y el feto. La violencia física o sexual que conlleva traumatismos abdominales puede provocar, según el tiempo de gestación, la pérdida del feto, el parto prema-turo o bajo peso en el recién nacido $(6,13,14)$.

El objetivo de este estudio fue determinar la prevalencia de violencia física, psicológica, emocional y sexual durante el embarazo y su asociación con el peso del hijo al nacer en una zona urbana marginal de San José, capital de Costa Rica.

\section{MATERIALES Y MÉTODOS}

La población de estudio estuvo constituida por todaslasmujeresembarazadasresidentesen la comunidad urbana marginal Finca San Juan que dieron a luz entre septiembre de 1998 y noviembrede 1999(118entotal). Enelmomento del estudio, esta zona era foco de la atención de instituciones que apoyan la labor social debido a sus características marginales. La Finca San Juan, deRincón GrandedePavas,SanJosé,Costa Rica, se clasificó como marginal, porque era un asentamiento precario donde la mayoría de las viviendas estaban en mal estado y las familias se abastecían de agua proveniente de un receptor común (de un tubo de cañería); la electricidad, la recolección de basura y el drenaje de aguas servidas, entre otros servicios, no eran adecuados.

A todas las mujeres residentes de ese poblado se les entrevistó en el hogar y se les aplicó un cuestionario diseñado para detectar si habían sufrido actos de violencia durante el embarazo. Esta encuesta contenía una pregunta directa sobre la violencia, además de otras que las mujeres debían responder aunque la respuesta directa acerca de abusos fuera negativa. Esto aumentó la sensibilidad del cuestionario, que había sido previamente validado en una población con características similares a las de la muestra del estudio. Las preguntas eran cerradas y estaban dirigidas a obtener información sociodemográfica acerca de la madre, su salud reproductiva, actos de violencia durante la gestación, hábitos maternos nocivos y los datos generales del control prenatal y del recién nacido. La información sobre la madre se corroboró con los expedientes clínicos de la institución adonde acudía para el control prenatal y del hospital donde ocurrió el parto. La información acerca del recién nacido se confirmó con el carné de salud del niño.

Las entrevistas se realizaron en un lapso no mayor de 72 horas después del parto (natural o por cesárea) o legrado. Este lapso se estableció de mutuo acuerdo con las madres. El tiempo promedio de la aplicación de la encuesta fue de 20,5 minutos (de 18,0 a 23,0 minutos; mediana: 21,0 minutos).
Los criterios de inclusión fueron dos: manifestar verbalmente la aceptación a participar en el estudio, una vez explicados los propósitos del mismo, y no tener impedimento físico para responder el cuestionario. Esta investigación fue aprobada por el Comité Ético Científico del Instituto Costarricense de Investigación y Enseñanza en Nutrición y Salud (INCIENSA).

Las madres que habían estado expuestas a agresiones fueron remitidas a los equipos básicos de atención integral de su comunidad y al departamento de trabajo social de la Clínica Central para la atención y seguimiento pertinentes.

Se estudiaron las siguientes variables:

Datos psicosociales. Embarazo deseado, embarazo planeado; si consideraba haber sido objeto de algún tipo de violencia durante el embarazo, identificación de quién ejercía la violencia, tipo de violencia (física, psicológica y emocional o sexual), momento de inicio de la violencia; características de la violencia física, zonas del cuerpo golpeadas, tipo de lesiones provocadas por los golpes; violencia sexual durante la gestación y lesiones provocadas; violencia psicológica y emocional y trastornos emocionales, incluida su frecuencia.

Datos demográficos y socioculturales. Edad, nacionalidad, estado conyugal, años de estudio (escolaridad), ocupación habitual, ingreso económico, hábitos de riesgo materno (tabaquismo, alcohol $\mathrm{u}$ otras drogas).

Salud reproductiva. Edad de inicio de la vida sexual, uso de anticonceptivos, control prenatal realizado, número de gestaciones y de partos previos, antecedente de hijos con BPN, estatura (en centímetros), aumento total de peso (en kilogramos) durante la gestación, enfermedades sufridas durante la gestación y espacio intergenésico (en meses). 
Datos sobre el recién nacido. Peso (en gramos) y clasificación según la edad gestacional.

Para los efectos de esta investigación se consideró como mujer agredida a toda aquella que así lo manifestara mediante respuesta verbal afirmativa.

La captura de los datos y el análisis estadístico de la información se realizaron mediante el programa Epi Info versión 5.0 (14). Se utilizó la prueba exacta de Fisher para detectar diferencias en cuanto a planificación familiar, asistencia al control prenatal, embarazo deseado, embarazo planeado, ocupación habitual de la madre, nacionalidad, estado conyugal (madre sola o con compañero), trastornos durante el embarazo (diabetes, hemorragias, hipertensión, anemia, asma, enfermedades renales), antecedente de hijo con BPN y hábitos de riesgo materno (tabaquismo, alcohol $\mathrm{u}$ otras drogas). Para el análisis de las variables continuas se aplicó la prueba de la $t$ de Student.

Se utilizó un modelo de regresión lineal múltiple para examinar las diferencias entre las medias del peso al nacer según la edad, los años de estudio maternos, la condición conyugal, el deseo del embarazo, los hábitos nocivos (tabaquismo, alcoholismo), el número de embarazos y partos previos, el intervalo intergenésico, la estatura materna, el aumento total de peso durante la gestación (en kilogramos) y la presencia de alguna enfermedad durante la misma. Se utilizó un modelo de regresión logística para medir el efecto directo de la violencia sobre el BPN (variable dependiente dicotomizada) y un método no paramétrico para calcular la fracción atribuible en las mujeres expuestas (14). Se utilizó un nivel de significación de 0,05.

\section{RESULTADOS}

\section{Características generales de la población estudiada}

Las gestantes representaron el 6\% de las 1964 mujeres con edades entre 10 y 43 años que vivían en la zona seleccionada en el período examinado. Todas las gestantes aceptaron participar en la investigación. En el cuadro 1 se presentan las características de la muestra total, del grupo de mujeres que no se sintieron agredidas y de las que refirieron haber sido víctimas de actos violentos.

La mayoría de las mujeres (78,0\%) eran costarricenses. Del total de 118 mujeres, 35 $(29,7 \%)$ estuvieron expuestas a algún tipo de violencia. Noventa y una $(77,1 \%)$ no sostenían relaciones clandestinas ni esporádicas. En 20 delas 27 que sostenían relaciones clandestinas, el embarazo se había iniciado durante un noviazgo corto; el resto de estas mujeres (cuatro nicaragüienses y tres costarricenses) fueron embarazadas por hombres casados o que vivían en unión libre con otra mujer. Las mujeres no casadas fueron significativamente más agredidas que las casadas $(P=0,037)$.

Aproximadamente $80,0 \%$ de los casos no tenían empleo remunerado y los ingresos mensuales de las que recibían retribución por trabajar tiempo completo eran de US\$ 100,00 a US\$ 200,00; el resto laboraba media jornada con ingresos mensuales entre US\$ 80,00 y US\$ 133,00 (al tipo de cambio de noviembre de 1999). Las ocupaciones que desempeñaban estas mujeres estaban acorde con su escolaridad; la mitad tenía de uno a seis años de estudio, 22\% completaron la primaria y solo $2,5 \%$ la secundaria. Las diferencias entre los promedios de ingresos mensuales y los años de estudio de las mujeres agredidas y las no agredidas no fueron significativas.

Con respecto al consumo de alcohol y al tabaquismo, tanto la prevalencia como la intensidad de ambos hábitos durante la gestación fueron bajas. Solo $5,0 \%$ de las mujeres refirieron haber ingerido licor (dos cervezas con $3,5^{\circ}$ de alcohol) en dos fines de semana y 14 mujeres $(11,9 \%)$ manifestaron haber fumado uno o dos cigarrillos semanales. En ambos casos lo hicieron durante el primer mes de embarazo. De esas 14 mujeres, una estuvo expuesta al humo del cigarrillo durante todo el embarazo, pues un hermano de su compañero, que padecía de trastornos psiquiátricos, fumaba de 10 a 14 cigarrillos por noche en una habitación separada por una cortina, a aproximadamente un metro de distancia de donde ella dormía. Tres de las 14 mujeres que fumaban también consumieron bebidas alcohólicas. No se encontró relación entre la frecuencia (intensidad) de estos hábitos y haber sido víctima de violencia.

\section{Características de la violencia durante el embarazo}

De las 35 mujeres que sufrieron actos de violencia, $33(94,3 \%)$ recibieron el maltrato de su pareja, una de su hermano mayor y la otra de su padre, es decir, todas fueron agredidas por hombres.

De las 35 mujeres agredidas, 23 $(65,7 \%)$ manifestaron que la violencia empezó tres años o menos antes de que se iniciara el embarazo y continuó durante el mismo, mientras que en 10 $(28,6 \%)$ se inició en el primer trimestre del embarazo, cuando el compañero se enteró de la situación. Es importante destacar que $6(60 \%)$ de estas últimas eran adolescentes y solteras. Las 2 restantes $(5,7 \%)$ refirieron haber sido agredidas cuando un familiar (hermano o padre) se enteró del embarazo; una de esas mujeres era adolescente y la otra adulta, ambas casadas. En estos casos, el abuso fue de tipo psicológico y emocional y se inició a finales del segundo trimestre del embarazo. El promedio de tiempo de vida en pareja fue de 3,4 $\pm 1,24$ años.

Las mujeres agredidas por sus compañeros manifestaron haber sufrido distintos tipos de abuso: predominó el psicológico o emocional $(86,9 \%)$, caracterizado por humillaciones verbales, gritos, abandono, negación de dinero para las necesidades básicas y amenazas de golpes. El abuso físico $(60,9 \%)$ consistió en golpes, empujones, cachetadas y mordiscos, mientras que el abuso sexual $(52,2 \%)$ se caracterizó por obligar a la mujer a realizar el coito. Aproximadamente $61,0 \%$ de las mujeres víctimas de la violencia de sus compañeros 


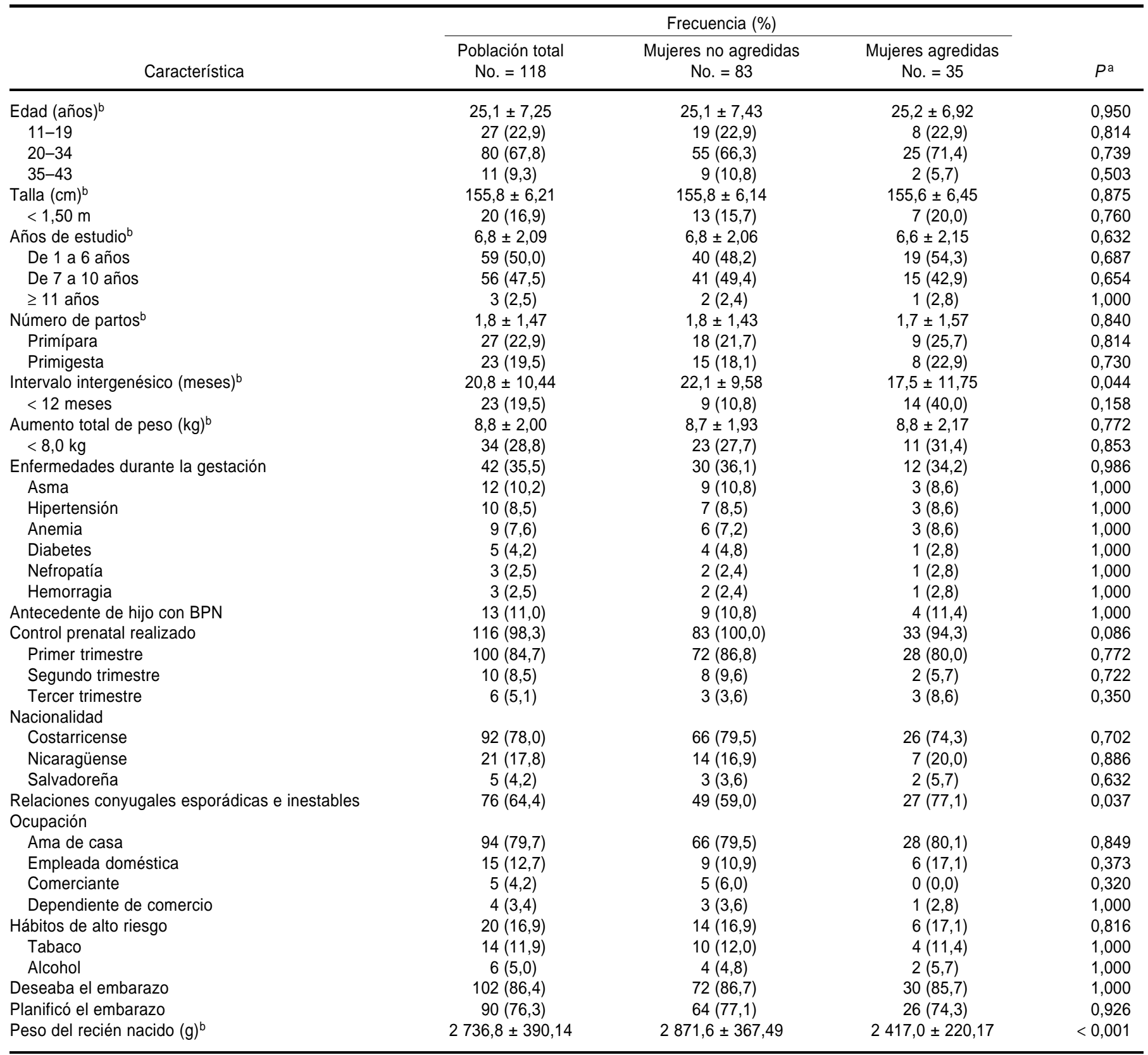

a Nivel de significación de 0,05.

b Media \pm desviación estándar.

manifestaron que la mayoría de las veces sucedió bajo los efectos del alcohol y en dos ocasiones también bajo los efectos de la marihuana y la cocaína.

La mayoría de las mujeres agredidas explicaron que el compañero les pegaba con los puños y las empujaba violentamente hacia las paredes $o$ contra el piso, aunque también hubo casos en que los golpes fueron propinados con objetos, como fajas, sogas y palos.

Ninguna mujer dijo haber sufrido lesiones de gravedad causadas por actos de violencia. Algunas de las que sufrieron abusos sexuales manifestaron que tuvieron dolor $\mathrm{y}$ sangrado vaginal. Las zonas del cuerpo golpeadas con hematomas e inflamaciones visibles fueron la cabeza (principalmente la cara), los hombros, los brazos, la espalda y los glúteos, y las mordeduras fueron en los senos, las orejas y los dedos de las manos. 


\section{La violencia y la salud reproductiva}

A pesar de que la mayoría de las mujeres estudiadas eran adultas, $19,5 \%$ de ellas estaban embarazadas por primera vez, $68,6 \%$ tenían de uno a tres embarazos previos y las restantes habían tenido de cuatro a siete embarazos. No se hallaron diferencias estadísticamente significativas con respecto al número de partos, al número de gestaciones previas ni al aumento de peso durante la gestación entre las mujeres agredidas y las que no habían sufrido agresiones. No obstante, las mujeres agredidas tuvieron intervalos intergenésicos significativamente más cortos que las demás $(P=0,044)$.

Con respectoalas enfermedades padecidas durante la gestación, aproximadamente una tercera parte de las mujeres tuvieron asma, hipertensión o anemia, sin diferencias significativas entre las mujeres que fueron agredidas y las que no lo fueron.

La mayoría de las mujeres tuvieron control prenatal durante el primer trimestre del embarazo, con excepción de dos nicaragüenses agredidas, y no se encontraron diferencias estadísticamente significativas entre las mujeres agredidas y las no agredidas. Alrededor de 86,0\% manifestaron que el embarazo fue deseado y $76,3 \%$, que lo planificaron, sin diferencias significativas entre los dos grupos de madres.

Los aspectos emocionales y sentimentales del embarazo fueron evaluados mediante siete categorías - feliz, apoyada, nerviosa y temerosa, deprimida (muy triste), subestimada, sin apoyo (sola) $\mathrm{y}$ enojada- $\mathrm{y}$ con cinco estados de intensidad según su frecuencia: nunca, raramente, a menudo, muy a menudo y siempre. El nivel de intensidad "muy a menudo" obtuvo la puntuación más alta en los dos grupos de mujeres en las siguientes categorías: "deprimida" (18,0\% en mujeres no agredidas frente a $57,1 \%$ en mujeres agredidas, $P<0,001)$, "subestimada" $(12,0 \%$ en mujeres no agredidas frente a $22,9 \%$ en mujeres agredidas, $P=0,226)$ y "nerviosa y temerosa" $(9,6 \%$ en mujeres no agredidas frente a $14,3 \%$ en mujeres agredidas, $P=0,524)$. El nivel de intensidad "siempre" para la categoría “apoyada" estuvo presente únicamente en las mujeres no agredidas (60,4\%), mientras que las mujeres agredidas se consideraron "apoyadas" solo "raramente" (5,7\%, P< $0,001)$. Se debe destacar que ninguno de los dos grupos de mujeres notificó haberse sentido "feliz" ni "enojada", y que los niveles de intensidad "nunca" y "a menudo" no fueron notificados para estas dos categorías. Las mujeres agredidas manifestaron sentirse significativamente más "deprimidas" y menos "apoyadas" que las no agredidas.

Por otro lado, la diferencia bruta entre los pesos promedio de los recién nacidos de las madres no agredidas y de las que sufrieron agresiones fue de 454,6 g ( $P<0,001)$. Este indicador se ajustó por edad, años de estudio, condición conyugal (casada o en unión libre, o relación esporádica), deseo del embarazo, presencia de hábitos nocivos (tabaquismo o etilismo), número de embarazos previos, número de partos anteriores, intervalo intergenésico, estatura, aumento total de peso durante la gestación y presencia de alguna enfermedad durante el embarazo, con una diferencia significativa de 449,4 gramos $(P<0,001)$.

Después de examinar la colinealidad entre las variables independientes (cuadro 2) se ensayaron varios modelos de regresión lineal múltiple (cuadros 3 y 4). Las variables que mostraron mayor asociación con el peso al nacer fueron la violencia y el aumento de peso durante la gestación. El modelo 2 (cuadro 4) resultó ser el más económico (parsimonious), ya que con menos variables logró explicar una

CUADRO 2. Resultados del análisis de colinealidad entre las variables independientes con significación estadística asociadas con el bajo peso al nacer ${ }^{a}$

\begin{tabular}{lcccccc}
\hline \multicolumn{1}{c}{ Variable } & $\begin{array}{c}\text { Condición } \\
\text { Violencia }\end{array}$ & $\begin{array}{c}\text { Edad } \\
\text { conyugal }\end{array}$ & $\begin{array}{c}\text { Número } \\
\text { de } \\
\text { (años) }\end{array}$ & $\begin{array}{c}\text { Aumento } \\
\text { total de } \\
\text { partos }\end{array}$ & $\begin{array}{c}\text { Intervalo } \\
\text { intergenésico } \\
\text { peso (g) }\end{array}$ & (meses) \\
\hline Violencia & 1,000 & 0,830 & 0,006 & 0,019 & 0,027 & 0,133 \\
Unión permanente & 0,830 & 1,000 & 0,030 & 0,027 & 0,039 & 0,163 \\
Edad (años) & 0,006 & 0,030 & 1,000 & 0,790 & 0,171 & 0,357 \\
Número de partos & 0,019 & 0,019 & 0,790 & 1,000 & 0,231 & 0,307 \\
$\begin{array}{l}\text { Aumento total de peso (g) } \\
\text { Intervalo intergenésico } \\
\text { (meses) }\end{array}$ & 0,027 & 0,039 & 0,171 & 0,231 & 1,000 & 0,316 \\
\hline
\end{tabular}

a Nivel de significación de 0,05. buena parte de las variaciones observadas en el peso al nacer $\left(R^{2}=46,1 \%\right)$.

Se observó que las mujeres agredidas fueron tres veces más propensas a tener hijos con BPN $(<$ $2500 \mathrm{~g})$ que las mujeres que no habían sido agredida s (desigualdad relativa $=3,3$; intervalo de confianza [IC] 95\%: $1,39$ a 8,$10 ; P=0,007)$. En las mujeres expuestas a la violencia la fracción atribuible del BPN fue de $69,7 \%$. Entre los recién nacidos de bajo peso, los neonatos con edad gestacional menor de 37 semanas fueron significativamente más pequeños que los nacidos después de 37 semanas de gestación para los clasificados como pequeños según la edad gestacional (razón de posibilidades $[\mathrm{RP}]=4,0 ; \mathrm{IC}$ 95\%: 1,6 a 10,$0 ; P=0,006)$. No se encontraron diferencias estadísticamente significativas entre los prematuros (edad gestacional < 37 semanas) y los recién nacidos con bajo peso $(\mathrm{RP}=$ 1,45; IC 95\%: 0,51 a 4,04; $P=0,593)$.

Por otro lado, el aumento total de peso durante la gestación de las madres de los recién nacidos con bajo peso fue como promedio de $7,9 \mathrm{~kg} \pm 1,76$ desviaciones estándar (DE), y el de las madres cuyos hijos nacieron con peso normal fue significativamente mayor $(9,1 \mathrm{~kg} \pm$ 2,20 DE; $P=0,002)$. También se observó un a diferencia significativa entre los promedios 
CUADRO 3. Modelo de regresión lineal múltiple, usando como variable dependiente el peso al nacer en gramos

\begin{tabular}{|c|c|c|c|c|c|c|}
\hline \multirow[b]{2}{*}{ Variable independiente } & \multicolumn{2}{|c|}{$\begin{array}{l}\text { Coeficientes no } \\
\text { estandarizados }\end{array}$} & \multicolumn{2}{|c|}{ Coeficientes estandarizados } & \multirow[b]{2}{*}{$P^{a}$} & \multirow[b]{2}{*}{$R^{2}(\%)$} \\
\hline & $\beta$ & $\begin{array}{l}\text { Error } \\
\text { estándar }\end{array}$ & $\beta$ & $\begin{array}{c}\text { Intervalo de } \\
\text { confianza de } 95 \%\end{array}$ & & \\
\hline (Constante) & 1843,14 & 710,57 & & 435,23 a 3251,04 & 0,011 & \\
\hline Edad (años) & $-3,46$ & 4,22 & $-0,064$ & $-11,84$ a 4,92 & 0,069 & 47,3 \\
\hline Aumento total de peso $(\mathrm{g})$ & 72,84 & 14,35 & 0,374 & 44,40 a 101,27 & $<0,001$ & \\
\hline Presencia de violencia & $-450,42$ & 60,05 & $-0,530$ & $-569,40 \mathrm{a}-331,43$ & $<0,001$ & \\
\hline Intervalo intergenésico (años) & 3,26 & 2,63 & 0,105 & $-1,95$ a 8,47 & 0,021 & \\
\hline
\end{tabular}

a Nivel de significación de 0,05 .

CUADRO 4. Tres modelos de regresión lineal múltiple usando como variable dependiente el peso al nacer en gramos

\begin{tabular}{|c|c|c|c|c|c|c|}
\hline \multirow[b]{2}{*}{ Modelo } & \multicolumn{2}{|c|}{$\begin{array}{l}\text { Coeficientes no } \\
\text { estandarizados }\end{array}$} & \multicolumn{2}{|c|}{ Coeficientes estandarizados } & \multirow[b]{2}{*}{$P^{a}$} & \multirow[b]{2}{*}{$R^{2}(\%)$} \\
\hline & $\beta$ & $\begin{array}{l}\text { Error } \\
\text { estándar }\end{array}$ & $\beta$ & $\begin{array}{c}\text { Intervalo de } \\
\text { confianza de } 95 \%\end{array}$ & & \\
\hline \multicolumn{7}{|l|}{ No.1 } \\
\hline (Constante) & 2261,94 & 147,62 & & 1969,48 a 2554,40 & $<0,001$ & \\
\hline Presencia de violencia & $-449,70$ & 59,87 & $-0,529$ & $-568,32$ a $-331,09$ & $<0,001$ & \\
\hline Aumento total de peso $(\mathrm{g})$ & 73,09 & 14,30 & 0,375 & 44,75 a 101,43 & $<0,001$ & 46,6 \\
\hline Intervalo intergenésico (meses) & 3,61 & 2,55 & 0,116 & $-1,45$ a 8,68 & 0,016 & \\
\hline \multicolumn{7}{|l|}{ No.2 } \\
\hline (Constante) & 2190,89 & 122,07 & & 1949,06 a 2432,73 & $<0,001$ & \\
\hline Presencia de violencia & $-453,47$ & 59,64 & $-0,533$ & $-571,62 \mathrm{a}-335,33$ & $<0,001$ & 46,1 \\
\hline Aumento total de peso $(\mathrm{g})$ & 72,77 & 14,28 & 0,373 & 44,48 a 101,70 & $<0,001$ & \\
\hline \multicolumn{7}{|l|}{ No.3 } \\
\hline (Constante) & 2191,39 & 122,25 & & 1949,23 a 2433,55 & $<0,001$ & \\
\hline Presencia de violencia & $-463,72$ & 59,06 & $-0,545$ & $-580,70 a-346,73$ & $<0,001$ & 45,0 \\
\hline Intervalo intergenésico (meses) & 2,65 & 2,29 & 0,085 & $-1,90$ a 7,20 & 0,025 & \\
\hline
\end{tabular}

a Nivel de significación de 0,05.

en meses de los intervalos intergenésicos de esos dos grupos de madres $(12,1 \pm 12,15$ meses en las mujeres agredidas frente a $18,8 \pm 12,18$ meses en las que no habían sido agredidas; $P=0,006)$.

\section{DISCUSIÓN}

El asentamiento de la Finca San Juan, ubicada en Rincón Grande de Pavas, dependencia de San José, capital de Costa Rica, se originó cuando el gobierno decidió buscar una solución parcial y provisional a las necesidades habitacionales de precaristas que habían invadido algunos terrenos. Los terrenos seleccionados para la construcción de viviendas son de suelo arcilloso, lo que dificulta el buen funcionamiento del sistema de letrinización. Las aguas negras y servidas se mantienen inadecuadamente y contaminan el ambiente. Además, las zonas verdes y áreas recreativas han ido cediendo sus espacios para la construcción de nuevas viviendas.

En el momento del estudio, el número de familias había aumentado de 836 a 1 300, con un total de 7800 habitantes (6 personas por familia como promedio); alrededor de la cuarta parte de la población eran mujeres en edad reproductiva, 34\% eran niños entre 0 y 9 años de edad, y $20 \%$ eran adolescentes.
Esta comunidad contaba con el apoyo de diversas instituciones sociales, como el Instituto Mixto de Ayuda Social (IMAS), el Instituto Nacional de Aprendizaje (INA), el Ministerio de Vivienda, el Ministerio de Educación Pública, el Ministerio de Salud, la Asociación de Desarrollo Comunal y de Salud, el Patronato Nacional de la Infancia (PANI), la Clínica de Pavas (COOPESALUD) y otras organizaciones no gubernamentales. La atención de la salud está fundamentalmente a cargo de la Clínica de Pavas.

Los resultados de esta investigación confirman que son hombres los que ejercen la violencia hacia las mujeres 
embarazadas (2, 3, 15-18), principalmente su pareja (19-21).

En Costa Rica, la información existente sobre la violencia hacia mujeres embarazadas no es sistemática ni continua. Una encuesta de opinión pública aplicada a 1200 personas mayores de 17 años en zonas urbanas del país (51,9\% mujeres y 48,1\% hombres) reveló la existencia de violencia física en $36,3 \%$ de los hogares, de violencia sexual en $21,4 \%$ y de violencia psicológica en $65,7 \%$. En esta encuesta no se especificó si hubo violencia durante el embarazo. ${ }^{4}$

El número de mujeres agredidas durante el embarazo que se encontró en esta investigación $(29,7 \%)$ es superior al informado por algunos autores extranjeros $(4,8-10,20,22,23)$ e inferior al informado en Brasil (1) y México (24). Estas diferencias pueden deberse al método empleado para recoger la información. McFarlene (25) encontró una prevalencia de violencia significativamente más baja $(7,3 \%)$ al utilizar un instrumento autoadministrado que cuando las entrevistas fueron realizadas por personal capacitado.

Está ampliamente documentado que entre las mujeres agredidas durante el embarazo hay mayor frecuencia de abortos espontáneos, de muerte intrauterina y neonatal y de hijos nacidos con BPN, lo que representa una limitación en las posibilidades de supervivencia del recién nacido (26). La clara asociación entre la violencia y el BPN coloca a la violencia como un problema de salud pública que requiere de intervención urgente por parte de las autoridades de salud (20, 27-30). Sin embargo, son pocos los estudios publicados en que se ha observado asociación entre la violencia durante la gestación y el peso del recién nacido como variable continua. En una investigación se informó que los hijos de madres no agredidas tenían mayor peso al nacer que

\footnotetext{
4 Centro Nacional para el Desarrollo de la Mujer y la Familia. Violencia intrafamiliar en Costa Rica. Encuesta de opinión en población urbana. Primeros resultados 1996. (Documento de trabajo reproducido localmente). Instituto Nacional de las Mujeres (INAMU). Informe estadístico. Delegación de la Mujer 2000. (Documento de trabajo reproducido localmente).
}

los de madres agredidas, con una diferencia bruta entre promedios de $229 \mathrm{~g}$ sin ajustes, y de $175 \mathrm{~g}$ después de hacer ajustes según educación materna, número de partos y antecedentes de adicciones (31). Otros autores encontraron diferencias brutas entre promedios de $133 \mathrm{~g}$ (27) y de $565,5 \mathrm{~g}$, con un descenso a $560 \mathrm{~g}$ una vez hechos los ajustes por edad y número de partos (24).

En la presente investigación, la diferencia bruta entre los pesos promedio de los recién nacidos de mujeres no agredidas y de mujeres agredidas fue de 454,6 gramos $(P<0,001)$. Al hacer ajustes por edad, escolaridad, condición conyugal, deseo del embarazo, presencia de hábitos nocivos (tabaquismo, alcoholismo), embarazos previos, número de partos, intervalo intergenésico, aumento total de peso durante la gestación, enfermedad durante la gestación y estatura, se observó una diferencia significativa de 449,4 gramos $(P<$ $0,001)$. Si bien el presente estudio fue de tipo transversal y exploratorio, la asociación significativa entre la violencia durante la gestación y el peso del recién nacido de madres que han sido agredidas constituye un signo de alarma que debe tomarse en cuenta de forma prioritaria debido al posible efecto de la violencia en la salud y la calidad de vida de la madre y el hijo.

Se debe destacar que la presente investigación es, hasta la fecha, la única en que se ha ajustado la diferencia entre los pesos promedio de los recién nacidos de las mujeres agredidas y de las no agredidas con mayor número de variables asociadas con el bajo peso al nacer $(8,9,11,15,29-31)$. Las variables independientes que se utilizaron en esta investigación para el ajuste de los pesos promedio, a diferencia del resto de los estudios, no solo fueron de naturaleza biológica (edad, número de embarazos y de partos previos, intervalo intergenésico, estatura, aumento total de peso y presencia de alguna enfermedad), sino también de naturaleza psico-sociocultural (escolaridad, condición conyugal, deseo del embarazo y hábitos nocivos durante la gestación) $(11,30,31)$.

Además, el presente estudio demostró que las variables más importantes asociadas con el bajo peso al nacer fueron la violencia sufrida por la madre (asociación directa) y el aumento de peso de la madre durante la gestación (relación inversa).

Por otro lado, se ha demostrado que vivir en un barrio marginal, como la comunidad donde se realizó esta investigación, se asocia con complicaciones durante el embarazo (3). Según otras investigaciones, la tensión psicológica está asociada con la depresión, tanto en las mujeres no embarazadas como en las embarazadas. En este estudio, el nivel de intensidad "muy a menudo" obtuvo el puntaje más alto en los dos grupos de mujeres en la categoría "deprimida". Además, se detectaron diferencias estadísticamente significativas entre las mujeres que se sentían deprimidas y las que se sentían apoyadas. Sobre este particular se ha planteado como hipótesis que la liberación de neurotransmisores como las catecolaminas - producto de la depresión y el estrés podría tener efectos directos en el feto al precipitar el parto prematuramente o causar hipoperfusión de la placenta con retraso del crecimiento fetal (32).

Por lo anterior, se recomienda que en futuros estudios se profundice en la evaluación del estado emocional de las mujeres mediante instrumentos estandarizados que midan los efectos del estrés postraumático, el desequilibrio psicológico-emocional y el apoyo psicosocial, lo que permitirá diseñar y desarrollar estrategias de intervención a favor de las mujeres en mayor riesgo.

El nivel socioeconómico pudo haber confundido la asociación entre la violencia y el peso al nacer por las implicaciones en la calidad de la dieta de las mujeres del estudio y por las diferencias en la exposición a otros riesgos sociales (hacinamiento, incesto, drogadicción). Sin embrago, la población estudiada fue homogénea en estos aspectos, con características sociodemográficas similares y propias de una comunidad urbana marginal.

Los especialistas que registraron los datos de los recién nacidos desconocían la condición de violencia de las mujeres, lo cual evitó sesgos durante el registro. Estos datos fueron 
obtenidos con básculas de similar sensibilidad, aunque con la limitación de que estos procedimientos no fueron supervisados por los investigadores, lo cual en todo caso, generaría un error de medición no diferencial que sesgaría el estimador hacia el valor nulo.

Algunos investigadores consideran que existen factores culturales que hacen ver la violencia como un hecho natural que se da dentro de una sociedad eminentemente machista (33). Esto influiría en la prevalencia de la violencia $y$, por ende, en sus efectos indeseables, y estos efectos pueden ser más intensos en la población adolescente por no haber alcanzado aún su completo desarrollo biológico, psicológico y social (1, 3, 21, 31, 33-35).

A partir de los principales hallazgos de esta investigación se propone:

1. Fomentar investigaciones interdisciplinarias y epidemiológicas during pregnancy in Rio de Janeiro, Brazil. Int J Gynecoly Obstet 2002;79 (3):269-277.

2. Anderson B, Marshak H, Hebbeler D. Identifying intimate partner violence at entry to prenatal care: clustering routine clinical information. J Midwifery Women's Health 2002; 47(5):353-359.

3. Heise L, Ellsberg M, Gottmoeller M. A global overview of gender-based violence. Int J Gynecoly Obstet 2002;78(Suppl. 1):S5-S14.

4. McFarlane J, Parker B, Soeken K. Abuse during pregnancy: associations with maternal health and infant birth weight. Nurs Res 1996; 45:37-42.

5. Heise L. Violencia contra la mujer: la carga oculta a la salud. Washington, D.C.: Banco Mundial, Organización Panamericana de la Salud; 1994.

6. Curry M, Harvey S. Stress related to domestic violence during pregnancy and infant birth weight. En: Campbell JC, ed. Empowering survivors of abuse. Thousand Oaks, California: Sage Publications; 1998. Pp. 98-108.

7. Kjellmer I, Liedholm M, Sultan B, Wennergren M, Wallin Götborg C, Thordstein $\mathrm{M}$. Long-term effects of intrauterine growth retardation. Acta Pediatr 1997;422(Suppl):8384 .

8. Berenson A, Wiemann M, Wilkinson G, Jones W, Anderson G. Perinatal morbidity associated with violence experienced by que utilicen técnicas cuantitativas y cualitativas para profundizar en la asociación entre la violencia hacia las mujeres embarazadas y el estado de salud del binomio madre-hijo.

2. Conformar grupos de expertos en este tema y desarrollar protocolos para la identificación temprana en las consultas prenatales de mujeres embarazadas agredidas.

3. Contemplar estos resultados en las normas existentes para la atención de las mujeres durante el embarazo, el parto y el puerperio, así como del recién nacido, con el objetivo de prevenir la aparición de casos de recién nacidos con bajo peso. Según los resultados de este estudio, si se lograra controlar la violencia durante el embarazo, en siete de cada diez mujeres expuestas se podría prevenir el nacimiento de niños con bajo peso.

Los protocolos de identificación de la violencia se han utilizado con buenos

\section{REFERENCIAS}

pregnant women. Am J Obstet Gynecol 1994;170(6): 1760-1769.

9. Dye T, Tollivert N, Lee R, Kenney C. Violence, pregnancy and birth outcome in Appalachia. Paediatr Perinat Epidemiol 1995;9:35-47.

10. Grimstad H, Schei B, Backe B, Jacobsen G. Physical abuse and low birthweight: a casecontrol study. Br J Obstet Gynaecol 1997;104: 1281-1287.

11. Bergonzoli G, Núñez H. Factores de la desnutrición intrauterina en neonatos a término. Colombia Médica 1997;28(4):167-176.

12. Murphy C, Schei B, Myhr T, Mont J. Abuse: a risk factor for low birth weight? CMAJ 2001; 164(11):1567-1572.

13. Rossenberg A, Stark E, Zahn M. Interpersonal violence: homicide and spouse abuse. En: Last $\mathrm{JM}$, ed. Public health and preventive medicine. Connecticut: Appleton-CenturyCrofts; 1998.

14. Dean A, Dean J, Brutus A. Epi-info versión 5. Epidemiología con microordenadores. Ginebra: USD Inc.; 1992.

15. Leung W, Kung F, Lam J, Leung T, Ho P. Domestic violence and postnatal depression in a Chinese community. Int J Gynecoly Obstet 2002;79(2):159-166.

16. Valladares E, Ellsberg M, Pena R, Hogberg U, Persson L. Physical partner abuse during pregnancy: a risk factor for low birth weight in Nicaragua. Obstet Gynecol 2002;100(4): 700-705. resultados en otros países (36-38). Para alcanzar esta meta se requiere impulsar un programa de capacitación y sensibilización del personal de salud (nutricionistas, ginecólogos, sociólogos, médicos generales o de familia, enfermeros, trabajadores sociales, pediatras y psicólogos) que está en contacto con las mujeres víctimas de actos de violencia en los diferentes niveles de atención, para que cuente con los elementos suficientes en el trabajo de detección de estos casos y la aplicación de procedimientos oportunos $(12,38,39)$.

Agradecimientos. Los autores agradecen profundamente a las 118 mujeres que participaron en este estudio; al Instituto Costarricense de Investigación y Enseñanza en Nutrición y Salud (INCIENSA) y al Fondo de las Naciones Unidas para la Infancia (UNICEF) de Costa Rica, el haber financiado esta investigación, y al Instituto Nacional de la Mujer (INAMU) el apoyo bibliográfico brindado.
17. Espinosa L, Osborne K. Domestic violence during pregnancy: Implications for practice. J Midwifery Women's Health 2002;47(5):305317.

18. Cloutier S, Martin S, Moracco K, Garro J, Clark K, Brody S. Physically abused pregnant women's perceptions of their relationships with their male partners. Women Health 2002; 35(2-3):149-163.

19. Jagoe J, Mangann E, Chauhan S, Morrisob J. The effects of physical abuse on pregnancy outcomes in a low-risk obstetric population. Am J Obstet Gynecol 2000;182:1067-1069.

20. Campbell J, Torres S, Ryan J, King C, Campbell DW, Stallings RY, et al. Physical and nonphysical partner abuse and other risk factors for low birth weight among full term and preterm babies: a multiethnic case control study. Am J Epidemiol 1999;150(7):714-726.

21. Kok J, Ouden AL den, Verloove-Vanhorick $P$, Brand R. Outcome of very preterm small for gestational age infants: The first nine years of life. Br J Obstet Gynecol 1998;105:162-168.

22. Webster J, Chandler J, Battistutta D. Pregnancy outcomes and health care use: effects of abuse. Am J Obstet Gynecol 1996;174:760-767.

23. Curry MA, Perrin N, Wall E. Effects of abuse on maternal complications and birth weight in adult and adolescent women. Obstet Gynecol 1998;92:530-534. 
24. Santiago R, Sanin-Aguirre LH. La violencia doméstica en el embarazo y su relación con el peso al nacer. Salud Publica Mex 1996;38:352362.

25. McFarlane J, Christoffel K, Bateman L, Miller B, Bullock L. Assessing for abuse: self-report nurse interview. Public Health Nurs 1991;8(4): 245-250.

26. Berenson A, Wiemann C, Wilkinson G, Jones W, Anderson G. Perinatal morbidity associated with violence experienced by pregnant women. Am J Obstet Gynecol 1995;172(5): 1644-1645.

27. McFarlane J, Parker B, Soeken K. Abuse during pregnancy: association with maternal health and infant birth weight. Nurs Res 1996; 45(1):37-42.

28. Peterson R, Gazmararian JA, Spitz AM, Rowley DL, Goodwin MM, Saltzman LE, et al. Violence and adverse pregnancy outcomes: a review of the literature and directions for future research. Am J Prev Med 1997;13:366-373.
29. Saigat S. Perception of health status and quality of life of extremely low-birth-weight survivors. Clin Perinatol 2000;27(2):403-418.

30. Samuelsen S, Bakketeig L. Does spousal physical abuse affect the outcome of pregnancy? Scand J Soc Med 1991;19(1):23-31.

31. Parker B, McFarlane J, Soeken K. Abuse during pregnancy: Effects on maternal complications and birth weight in adult and teenage women. Obstet Ginecol 1994;84(3):323-328.

32. Zuckerman B, Amaro H, Bauchner H, Cabral $\mathrm{H}$. Depressive symptoms during pregnancy: relationships to poor health behavior. Am J Obstet Gynecol 1989;160:1107-1111.

33. Díaz C, Sotelo J. Domestic violence in Mexico. JAMA 1996;275(24):1937-1941.

34. Núñez H, Rojas A. Revisión conceptual y comportamiento del embarazo en la adolescencia en Costa Rica, con énfasis en comunidades urbanas pobres. Rev Costarric Salud Publica 1998;7(13):38-52.

35. Campbell J, Poland M, Waller J, Ager J. Correlates of battering during pregnancy. Res Nurs Health 1992;15:219-226.
36. Reid A, Biringer A, Carroll J, Midmer D, Wilson L, Chalmers B, et al. Using the ALPHA form in practice to assess antenatal psychosocial health. CMAJ 1998;159(6):677-684.

37. Levitt C. Canadian perinatal surveillance system. Can Fam Physician 1998;44:583.

38. Edin K, Hogberg U. Violence against pregnant women will remain hidden as long as no direct questions are asked. Midwifery 2002; 18(4):268-278.

39. Parker B. Untitled. J Midwifery Women's Health 2002;47(5):401-402.

Manuscrito recibido el 25 de marzo de 2002. Aceptado para publicación, tras revisión, el 3 de marzo de 2003.
ABSTRACT

Physical, psychological, emotional, and sexual violence during pregnancy as a reproductive-risk predictor of low birthweight in Costa Rica
Objective. To determine the prevalence of physical, psychological, emotional, and sexual violence during pregnancy and the association that that violence has with low birthweight.

Methods. For 118 women who gave birth between September 1998 and November 1999 we explored the violence that they had suffered during their pregnancy. All the women were residents of Finca San Juan, a low-income urban settlement in the Rincón Grande de Pavas section of San José, Costa Rica. We used a previously validated questionnaire with closed questions. A multiple linear regression model was used to adjust the average weights of the newborns according to the characteristics of the mother: age, years of schooling, marital status, desire for the pregnancy, harmful habits (smoking, drinking alcohol), number of previous pregnancies and childbirths, birth interval, physical stature, total increase in weight during the pregnancy, and illnesses during the gestation. A logistic regression model was used to measure the direct effect of violence on low birthweight, and a nonparametric method was used to calculate the attributable fraction among the exposed women.

Results. The newborns of the mothers who suffered acts of violence weighed on average $449.4 \mathrm{~g}$ less that the newborns of the women who had not been exposed to acts of violence $(P<0.001)$. The mothers who suffered acts of violence were three times as likely to have a newborn with low birthweight ( $95 \%$ confidence interval: 1.39 to 8.10 ). The variables that were most closely associated with low birthweight were violence suffered by the mother (direct association) and the mother's weight gain during pregnancy (inverse relation).

Conclusions. Our results indicate the need to investigate this subject more deeply, to train health workers concerning violence toward women as a reproductive-risk factor, and to form groups of experts on this subject to develop specialized protocols for the early identification of pregnant women subject to violence. 